$\xi=$ 줄

\title{
Comparative studies between herbal toothpaste (dantkanti) and non-herbal tooth paste
}

\author{
Kuldeep Singh $^{1 *}$, Pooja Singh ${ }^{2}$, Gurpreet Oberoi ${ }^{3}$ \\ ${ }^{1}$ Head of the Dental clinic and research centre, Patanjali Yogpeeth, Haridwar, India \\ ${ }^{2}$ Dental Clinic, bahadrabad, Haridwar, India \\ ${ }^{3}$ Dental clinic and research centre, Patanjali Yogpeeth, Haridwar, India \\ *Corresponding author E-mail: kuldeep_manipal@yahoo.co.in
}

\begin{abstract}
The purpose of the paper is to highlight the advantages of using the herbal tooth paste with that of conventional paste. The approach is to make use of naturally available herbs such as Akarkara (anacyclus pyrethrum), Neem (azadirachta indica), Babool (acacia arabica), Haldi (curcuma longa) etc to make herbal paste. These herbal mixture acts as antimicrobial agents. The clinical study is carried out with three parameters - Debris index, Calculus index and Gingival bleeding index. These parameters were compared over conventional paste. We have carried out studies with 100 people of different age groups ranging from 25 to 50 years covering both men and women. The statistical t-test is carried out between the unpaired two groups of herbal and non-herbal pastes. The results shows that the group - A (herbal) has significantly lower debris, calculus and gingival bleeding index value. The debris index and calculus index have $\mathrm{p}<0.001$, the oral hygiene index also has the $\mathrm{p}<0.001$, which is significant and the gingival bleeding index has the $\mathrm{p}<0.05$.

The conclusion is that the herbal Dantkanti toothpaste is more efficient in maintenance of oral hygiene and gum bleeding as compared to the non-herbal toothpaste.
\end{abstract}

Keywords: Calculus; Debris; Gingivitis; Herbal Toothpaste; Oral Hygiene

\section{Introduction}

The aim of this study is to assess the efficacy of Herbal toothpaste (Dantkanti) as compared to non-herbal toothpaste on the oral hygiene \& gum bleeding with the help of different types of dental indices including OHI-S \& GI index. It is a two group comparative study. Food debris are white small particles on the teeth, can be easily rinsed off. Dental plaque is a thin film of bacteria that sticks to teeth, yellow in colour can't be rinsed off, removed only by brushing \& flossing. Calculus is calcified plaque, hard, brownish or darkish in colour only removed by scaling. If plaque is not removed regularly by tooth brushing and flossing, it hardens to create calculus (also known as tartar). Dental calculus is made from mineralized plaque and other deposits on the teeth surface. It is so hard and attached to teeth very tight. General tooth brushing process cannot remove it. There has been closer relationship between tartar, calculus and periodontal diseases. Calculus can provide a good place for plaque attaching and bacteria growth. Calculus can absorb so much bacterial toxins that irritate the soft tissue, gingival edema and cause bleeding gums.

Dental Plaque deposit on teeth is a concern because of its cosmetic and pathogenic nature. Presence of plaque may be the culprit for dental caries, gingivitis, periodontal problem, and halitosis. Many mechanical aids are used worldwide to remove or control plaque, including toothbrushes, dental floss, and mouth rinses, and dentifrices (Barnes VM, Ritcher R, De Vizio W, 2010). Mechanical plaque removal is one of the most accepted methods of controlling plaque and gingivitis. Mechanical plaque control is time consuming and some individuals may lack motivation for these procedures (Mullaly BH et al, 1995). There has been a search for years for chemical agents that could supplant patient- dependent mechanical plaque control and thus reduce or prevent oral disease Several chemical preventive agents have beneficial effects in the control of plaque and to reduce or prevent oral disease. Hence, various chemical formulations were tried in dentifrices (George $\mathrm{J}$ et al, 2009). Chemicals, mainly triclosan and chlorhexidine have been added in mouth rinses and dentifrices to prevent plaque and gingivitis. But some of these substances show undersirable side effects such as tooth staining and altered taste (Barnes VM, Ritcher R, De Vizio W, 2010 and De Oliveira SM et al, 2008). This led to paying increased attention on using natural ingredients in herbal dentifrices. Herbal ingredients have several benefits.Commonly used herbal ingredients are Akarkara (anacyclus pyrethrum), Neem (azadirachta indica), Babool (acacia arabica), Haldi (curcuma longa)etc. Neem have antibacterial (Nayak A et al, 2011) and anti-inflammatory properties. Neem also have anti caries properties (Prashant GM et al, 2007 and Packia Lekshmi NCJ et al, 2012). The antimicrobial effects of neem have been reported against S.mutans and S.faecalis (Siswomihardjo W, 2007 and Almas K, 1999). Dried chewing sticks of neem shows maximum antibacterial activity against S.mutans(Chava VR et al, 2012 ).

There are limited studies available regarding the efficacy of herbal dentifrices hence the present study was undertaken to assess their effect on oral hygiene and gingival bleeding.

\section{Method and material}

In this study 100 subjects were screened from our Dental Clinic, Patanjali Yogpeeth, Haridwar their ages ranged from 25 to 55 
years. Ethical Approval was obtained from the institutional review board and signed consent form was obtain from the subjects

\subsection{Inclusion criteria}

Subjects who met the following inclusion criteria were included in this study: Having a minimum of 20 teeth, good general health, presence of established gingivitis, willing to give a written informed consent.

\subsection{Exclusion criteria}

Exclusion criteria were: Presence of advanced periodontitis, probing depth $>4 \mathrm{~mm}$ subjects under antimicrobial therapy, , pregnant women, using orthodontic appliances, having used mouth rinse containing chemical agents in previous 3 months, and having a history of allergy to toothpastes.

\section{Design}

The study was designed as a two group comparative study. The subjects were divided into two groups each having 50 subjects. Group A subjects are using Dantkanti Tooth paste for more than one month and group B subjects are using any other non-herbal toothpaste

The subjects are divided into two groups

GROUP-A: The subject of this group is using Dantkanti Tooth Paste for more than one month. The age of subjects is between 25 to 50 years. Number of subjects in the group is 50 .

GROUP-B: The subject of this group is using other non-herbal tooth pastes. The age of subjects is between 25 to 50 years. Number of subjects in the group is 50 .

\subsection{Index examination}

a) OHI-S: This index is measured to access the oral hygiene of the patients by examining the debris index and calculus index.

b) Gingival Index (GI): This index is examined to assess the health of Gingiva by assessing their bleeding on probing by periodontal probe.

\section{Observations \& results}

Data analysis done by t-test.The group A has significantly lower debris $(\mathrm{P}<0.001)$, calculus $(\mathrm{P}<0.001)$ and gingival index value $(\mathrm{P}<0.05)$ as compared to group $\mathrm{B}$. In this study the average debris index and calculus index of group A is less than the group B. It means that the herbal toothpaste (Dantkanti Toothpaste) is helpful in maintenance of oral hygiene by reducing the debris and calculus. The average gingival index of group $\mathrm{A}$ is less than the group B it means that the herbal content of toothpaste have antibacterial effect and hence reducing the inflammation of gingiva and bleeding from gums.

The Assessment is done using following Questionnaire

\subsection{Simplified oral hygiene index (OHI-S)}

As per (Soben Peter, 2004), the simplified form of Oral Hygiene Index (OHI -S). This index consist of two parts:

\subsection{Debris index - simplified (DI - S)}

Oral debris is the soft foreign matter loosely attached to the teeth. It consists of mucin bacteria and food and varies in color from grayish white to green or orange. This part of the questionnaire is to measure the surface area covered by debris. It is estimated by running the side of the explorer along the tooth surface examined. Then the examiner will measure as per the scale proposed from 0 to 3 debris deposition on a scale of 4 options.

\subsection{Calculus index-simplified (CI-S)}

Dental calculus is defined as deposit of inorganic salts composed primarily of calcium carbonate and phosphate mixed with food debris; bacteria and desquamated epithelial cells .There are two main types of calculus which are differentiated primarily by location on the tooth in relation to the free gingival margin. It may be Supragingival calculus or Sub gingival calculus. Then the examiner will measure as per the scale proposed from 0 to 3 calculus deposition on a scale of 4 options

\subsection{Gingival index (GI)}

As per(Soben Peter, 2004) for assessing the severity of gingivitis and its location in four possible areas by examining the qualitative changes(severity of the lesion)of the gingival soft tissue.The GI does not take into account of periodontal pocket depth bone loss or any other quantitative changes of the periodontium.

No prophylaxis was undertaken before the commencement of the study and no attempt was made to modify the volunteers oral hygiene habits.

\section{Details about the herbal toothpaste compo- sition}

The Herbal toothpaste used in this study is Dantkanti dental cream ,a product of Patanjali Ayurved Limited Haridwar, The main herbal ingredient of this paste is Akarkara (anacyclus pyrethrum), Neem (azadirachta indica) ,Babool (acacia arabica) ,Pudina (menthe spicata) ,Long bud (syzygium aromaticam), Tomar (xanthoxylum alatum), Haldi (curcuma longa),Pilu (salvadora persica) ,Bakul (mimusops elengi), Vidang (Embelia ribes) etc. The above herbal ingredient have the known effect of antibacterial, anti inflammatory and anti cariogenic properties.

\section{Data extraction}

It is given by the formula; OHI-S = DI-S+CI-S, where abbreviations are defined elsewhere in the paper

\subsection{Debris index scoring criteria}

The scoring criteria are given by;

1) No debris or stain present.

2) Soft debris covering not more than one third of the tooth surface or presence of extrinsic stains without debris regardless of surface area covered.

3) Soft debris covering more than one third but not more than two thirds of the exposed tooth surface.

4) Soft debris covering more than two thirds of the exposed tooth surface.

\subsection{Caculus index scoring criteria}

The scoring criteria are given by;

1) No calculus present

2) Supra gingival calculus covering not more than one third of the exposed tooth surface

3) Supra gingival calculus covering more than one third exposed tooth surface or the presence of individual flecks of sub gingival calculus around the cervical portion of the tooth or both .

4) Supra gingival calculus covering more than two third of the exposed tooth surface or a continuous heavy band of sub gingival calculus around the cervical portion of the tooth or both.

The DI-S and $\mathrm{CI}-\mathrm{S}$ values range from 0 to 3 . This is shown in Table 1. 
Table 1: Grading For DI-S and CI -S

\begin{tabular}{lll}
\hline S1.No. & Range & Grading \\
1 & 0.0 to 0.6 & Good \\
2 & 0.7 to 1.8 & Fair \\
3 & 1.9 to 3 & Poor \\
\hline
\end{tabular}

The OHI-S value ranges from 0 to 6 given in Table 2 .

Table 2: Grading for OHI-S

\begin{tabular}{lll} 
& \multicolumn{2}{l}{ Table 2: Grading for OHI-S } \\
\hline S1.No. & Range & Grading \\
\hline 1 & 0.0 to 1.2 & Good \\
2 & 1.3 to 3.0 & Fair \\
3 & 3.1 to 6 & Poor \\
\hline
\end{tabular}

\subsection{Scoring criteria for gingival index}

The scoring criteria for GI $-\mathrm{S}$ are given by;

1) Absence of inflammation / normal gingiva

2) Mild inflammation, slight change in colour, slight edema, no bleeding on probing

3) Moderate inflammation, moderate glazing, redness, edema and hypertrophy, bleeding on probing

4) Severe inflammation, marked redness and hypertrophy ulceration tendency to spontaneous bleeding

The numerical scores of the gingival index may be associated with varying degrees of clinical gingivitis as given in Table 3 .

Table 3: Scoring Rates

\begin{tabular}{|c|c|c|}
\hline S1. No. & Gingival score & Condition \\
\hline 1. & $0.1-1$ & Mild \\
\hline 2. & $1.1-2$ & Moderate \\
\hline 3. & $2.1-3.0$ & Severe \\
\hline
\end{tabular}

\section{Data analysis and results}

The data of group A and Group B was compared by unpaired t-test and its average values are shown in Tables 4 and 5.

Table 4: Average Values for $\mathrm{OHI}$ and GI

\begin{tabular}{lll}
\multicolumn{3}{c}{ Table 4: Average Values for OHI and GI } \\
\hline Indicators & Group A & Group B \\
\hline Average OHI $-\mathrm{S}$ & $1.26 \pm 0.62$ & $2.50 \pm 0.85$ \\
Average GI & $1.32 \pm 0.44$ & $1.52 \pm 0.33$ \\
\hline
\end{tabular}

Table 5: Average Values for Debris Index and Calculus Index

\begin{tabular}{lll}
\hline Indicator & Group A & Group B \\
\hline Average DI - S & $0.80 \pm 0.34$ & $1.21 \pm 0.43$ \\
Average CI- S & $0.46 \pm 0.36$ & $1.28 \pm 0.47$ \\
\hline
\end{tabular}

The data of both groups A and B is compared by unpaired t-test shows that the group A has significantly lower debris $(\mathrm{p}<0.001)$, calculus $(\mathrm{p}<0.001)$ and gingival index value $(\mathrm{p}<0.05)$ as compared to group $\mathrm{B}$.

\section{Discussion}

Lately there has been a growing interest in natural products the principal ingredients of the toothpaste used in this study may possess many medicinal properties however data pertaining to the substantivity of these products is spares it is imperative that clinical trials verify the efficacy of any new product. Dental plaque is the main culprit for gingival inflammation and dental caries. Chronic gingival inflammation may lead to tissue destruction and if left untreated may progress onto the more destructive stages of periodontitis (Barnes VM, Ritcher R, De Vizio W, 2010). Hence plaque and gingivitis control helps in maintenance of healthy oral cavity. This can be achieved effectively by mechanical plaque control using tooth brush and medicated toothpaste (Willerhausen B Gruber I Hamm G, 1991) have shown that the $\mathrm{pH}$ of the total saliva was significantly displaced into the alkaline range by the use of herbal products whereas in this study, there was no statistically significant difference in the salivary $\mathrm{pH}$ range in the test group. Salivary $\mathrm{pH}$ in the control group was found to be displaced more towards the acidic range (Willerhausen B Gruber I Hamm G, 1991).

In this study the average debris index and calculus index of group $\mathrm{A}$ is less than the group B.It means that the herbal toothpste Dantkanti is helpful in maintenance of oral hygiene by reducing the debris and calculus. The average debris index (DI-S)and calculus index (CI-S) of group A is $0.80 \pm 0.34$ and $0.46 \pm 0.36$ respectively while of group B is $1.21 \pm 0.43$ and $1.28 \pm 0.47$. The value of oral hygiene index (OHI-S) for group A and group B is $1.26 \pm 0.62$ and $2.50 \pm 0.85$ repectively. As it is clear from the above data that the average value of debris and the calculus index of group A is lesser than the group B ,and hence the oral hygiene of group A is better than the group B.

The average gingival index of group A is less than the group B The average value of gingival index of group A and group B is $1.32 \pm 0.44$ and $1.52 \pm 0.33$ respectively. It means that the herbal content of toothpaste have antibacterial effect and hence reducing the inflammation of gingiva and bleeding of gums.

In the analysis by t-test the group A has significantly lower debris, calculus and gingival index value. The debris index and calculus index have $\mathrm{P}<0.001$.the oral hygiene index also has the $\mathrm{P}<0.001$ which is significant, and the gingival index has the $\mathrm{P}<0.05$. All these values are significantly lower. It proves that the herbal Dantkanti toothpaste is more efficient in maintenance of oral hygiene and gum bleeding as compared to the non-herbal toothpaste The control group composed of fluoride and triclosan ingredients in toothpaste. Triclosan is an antimicrobial agent with wellestablished safety and efficacy (Radafshar G, Mahboob F, Kazemnejad E, 2010). Fluoride has anti-caries effect, but some of the constituents of the conventional toothpastes have undesirable side effects like staining and taste alterations. Hence, natural products with added benefits are advised for use.[9] Several studies have proven the anti-plaque and anti-gingival effects of herbal toothpaste, which were comparable to those of conventional toothpastes (George J et al, 2009, Radafshar G, Mahboob F, Kazemnejad E, 2010, Ozaki F et al, 2006, Sushma S, Nandlal B, Srilatha KT, 2011, Mazumdar M et al, 2013).

Several other studies (George $\mathrm{J}$ et al, 2009, Radafshar G, Mahboob F, Kazemnejad E, 2010, Mazumdar M et al, 2013, Mateu FA et al, 2008 ) also proves the validity of our observations on the effectiveness of herbal dentifrices in plaque control, compared to the conventional one..

The primary purpose of this study was to evaluate the efficacy of herbal dentifrice in maintenance of oral hygiene and gingivitis. No adverse reactions to dentifrices products were observed during the trial in the present study.This is in agreement with reports by (Ozaki F et al,) oliveira and ozaki et al. 2006, De Oliveira SM et al, 2008. The present study has proved that herbal dentifrices do not cause any adverse effects on the oral cavity and are effective in maintenance of oral hygiene and gingivitis, as that of fluoridated non - herbal dentifrice. Several studies have proven the medicinal values of herbal products (Nayak A et al, 2011, Mazumdar M et al, 2013). Hence medicated herbal toothpaste can be safely used to control plaque and gingivitis

The present study has proved that herbal dentifrices do not cause any adverse effects on the oral cavity and are effective in reduction of plaque and gingivitis, as that of fluoridated non-herbal dentifrice. Several studies have proven the medicinal values of herbal products (Nayak A et al, 2011, Mazumdar M et al, 2013). Hence, medicated herbal toothpastes can be safely used to control plaque and gingivitis. Further long-term studies are required to prove their effectiveness.

\section{Conclusions}

The group A using the herbal Dantkanti toothpaste is effective in terms of reduction of debris, caculus and Gingival index factor is statistically significantly lower. No adverse reactions of dentifrices products were observed during the trial and therefore it may be concluded that clinically herbal dentifrices are more effective than 
non - herbal paste in the control of oral hygiene and gingival ailments

Finally, it may be concluded that the herbal toothpaste (Dantkanti) is effective when compared to the conventional toothpaste in maintenance of oral hygiene and gum bleeding.

\section{Acknowledgements}

The authors gratefully acknowledge the blessings and support received from Acharya Balkrishna ji, Patanjali Yogpeeth, Haridwar in getting this paper to see the day light. Our thanks are also due to the support of my other colleagues working in the Patanjali Yog peeth especially, Dr. G. Paran Gowda.

\section{References}

[1] Barnes VM, Ritcher R, De Vizio W. Comparision of the short term antiplaque/ antibacterial efficacy of two commercial dentrifrices. J Clin Dent.2010, 21:101-4. [PubMed]

[2] Mullaly BH, James JA, Coulter WA, Linden Gj the efficacy of a herbal based tooth palste on the control of plaque and gingivitis. J Clin Periodontal 1995;22.686-9

[3] George J, Hegde S, Rajesh KS, Kumar A. The efficacy of a herbalbased toothpaste in the control of plaque and gingivitis: A clinicbiochemical study. Indian J Dent Res. 2009; 20:480-2. [PubMed] http://dx.doi.org/10.4103/0970-9290.59460.

[4] Nayak A, Ranganathan N, Sowmya GB, Kishore B, Kudalkar M Evaluation of antibacterial and anticandidial efficacy of aqueous and alcoholic effect of neem (Azadirachta Indica) An Invitro study. Int J Res Ayurveda Pharm. 2011; 2:230-5.

[5] De Oliveira SM, Torres TC, Pereira SL, Mota OM, Carlos MX. Effect of dentifrice containing Aloe vera on plaque and gingivitis control. A double-blind clinical study in humans. J Appl Ora Sci. 2008; 16:293-6. [PubMed] http://dx.doi.org/10.1590/S167877572008000400012.

[6] Prashant GM, Chandu GN, Murulikrishna KS, Shafiulla MD. The effect of mango and neem extract on four organisms causing dental caries Streptococcus Mutans Streptococcus Salivavius, Streptococcus MitisAnd streptococcus Sanguis: An in Vitro study. Indian J Dent Res. 2007; 18:148-51. [PubMed] http://dx.doi.org/10.4103/0970-9290.35822.

[7] Packia Lekshmi NCJ, Sowmia N, Viveka S, Raja Brindha J, Jeeva $\mathrm{S}$. The inhibiting effect ofAzadirachta indica against dental pathogens. Asian J Plant Sci Res. 2012;2:6-10

[8] Siswomihardjo W, Sunarintyas SB, Nishimura M, Hamada T. The difference of antibacterial effect of neem leaves and stick extract. Int Chin J Dent. 2007; 7:27-9.

[9] Almas K. The antimicrobial effects of extracts of Azadirachta Indica (Neem) and Salvadora Persica(Arak) chewing sticks. Indian J Dent Res. 1999; 10:23-6. [PubMed]

[10] Willerhausen B Gruber I Hamm G The influence of herbal ingredients on the plaque index and bleeding tendency of gingival. J Clin Dent $1991 ; 2 ; 75-8$.

[11] Radafshar G, Mahboob F, Kazemnejad E. A study to assess the plaque inhibitory action of herbal-based toothpaste: A double blind controlled clinical trial. J Med Plants Res. 2010; 4:1182-6.

[12] Ozaki F, Pannuti CM, Imbronito AV, Pessotti W, Saraiva L, de Freitas NM, . Efficacy of a herbal tooth paste on patients with established gingivitis--a randamised controlled trial. Braz Oral Res. 2006; 20:172-7.[PubMed http://dx.doi.org/10.1590/S180683242006000200015.

[13] Sushma S, Nandlal B, Srilatha KT. A comparative evaluation of a commercially available herbal and non- herbal dentifrice on dental plaque and gingivitis in children-A residential school based oral health programme.j Dent Oral Hyg.2011;3;109-13

[14] Mazumdar M, Chatterjee A, Majumdar S, Mahendra C, Patki PS. Evaluation of the safety and efficacy of complete care herbal toothpaste in controlling dental plaque, gingival bleeding and periodontal diseases. J Homeop Ayurv Med. 2013; 2:1-5.

[15] Chava VR, Manjunath SM, Rajanikanth AV, Sridevi N. The efficacy of neem extract on four microorganisms responsible for causing dental caries viz Streptococcus Mutans, Streptococcus Salivarius, Streptococcus Mitis and Streptococcus Sanguis: An in Vitro study. J Contemp Dent Pract. 2012;13:769-72.[PubMed]

[16] Mateu FA, Boneta AE, De Vizio W, Stewart B, Proskin HM. A clinical investigation of the two dentifrices for controlling estab- lished supragingival plaque and gingivitis. J Clin Dent.2008;19:8594. $\{$ PubMed $\}$

[17] Soben Peter, 2004, Essentials of Preventive and Community Dentistry, second edition, Arya Publishing House, New Delhi, India, page. No. 136-140.

[18] Soben Peter, 2004, Essentials of Preventive and Community Dentistry, second edition, Arya Publishing House, New Delhi, India, page. No. 153-155. 